\title{
USING OF WATERJET TECHNOLOGY FOR CUTTING ALUMINUM ALLOY
}

\author{
Tomáš Kroupa, Milan Daňa \& Miroslav Zetek
}
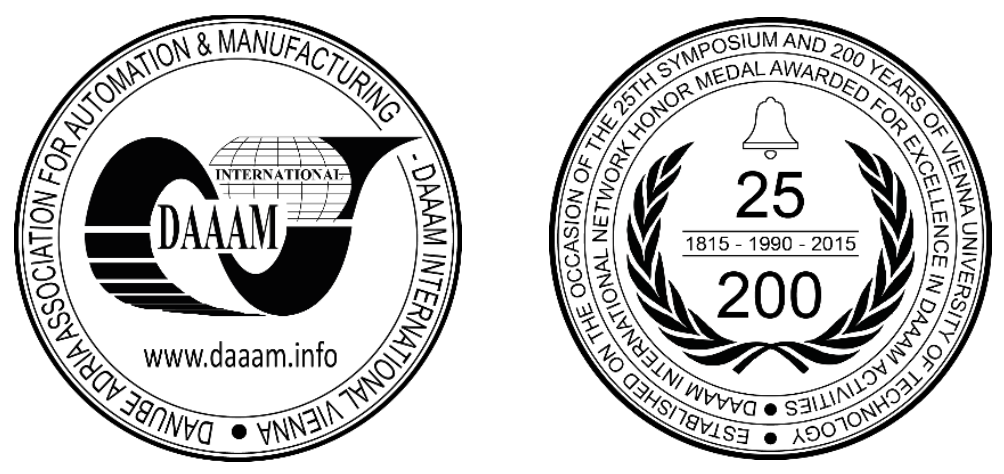

This Publication has to be referred as: Kroupa, T[omas]; Dana, M[ilan] \& Zetek, M[iroslav] (2017). Using of Waterjet Technology for Cutting Aluminum Alloy, Proceedings of the 28th DAAAM International Symposium, pp.0486-0492, B. Katalinic (Ed.), Published by DAAAM International, ISBN 978-3-902734-11-2, ISSN 1726-9679, Vienna, Austria DOI: $10.2507 / 28$ th.daaam.proceedings.068

\begin{abstract}
This paper presents an analysis of the impact of AWJ Machining on the surface of Aluminium (AlMg3) parts. Samples of Aluminium are subjected to AWJ surface machining. The essence of this cutting method is the grinding of a material by using pressure from a waterjet. The jet is generated in the cutting head and terminates in the nozzle. A range of machining parameters is considered, including feed rate, cut quality, speed, and static shot. These samples are then subject to surface analysis. This analysis allows conclusions to be drawn with regard to optimal machining conditions for cut quality improvement. This experiment provided a practical verification of the theoretical assumption. The practical part with the cut samples should serve mainly to provide a better idea and easier understanding of this technology. Samples which were cut using AWJ were documented and compared.This experiment also shows that it is necessary to pay attention to ensuring the correct cutting parameters for a specific application, because even here can be seen the eternal duel between quantity versus quality.
\end{abstract}

Keywords: Unconventional machining technology; cutting material by waterjet; high pressure waterjet; abrasive material cutting

\section{Introduction}

Waterjet cutting technology uses the kinetic energy of a high pressure and high speed water jet for cutting material. Abrasive methods also use the kinetic energy of abrasive particles, which makes a significant difference. Material is cut by high speed erosion by abrasive particles which are concentrated into a thin ray over a small surface area. The speed of this beam is $600-900 \mathrm{~m} / \mathrm{s}$. It is called "hydroabrasive erosion" which is induced by a high speed hydroabrasive beam. The beam is the cutting tool in this type of technology. The particle removal of the cut material is micro-dimensional. This erosive removal goes on at practically non-elevated temperatures and therefore there is no thermal deformation around the cut. This means it is possible to cut materials that could not be divided by other methods.

Waterjet technology uses a mechanical-physical method of material removal. A very thin stream of water which has a high speed acts on the workpiece. This energy causes the beam to pass through the material, thereby machining it. 


\section{Methods of water jet cutting}

Waterjet cutting is divided in two main methods. One method is waterjet machining, which is pure waterjet cutting. The second method is abrasive waterjet cutting (abbreviated AWJ) - which uses the addition of an abrasive medium. Both methods are similar, but they differ in the added abrasive material. In both of these methods, the cut material can be placed underwater and the device is similar. In both methods there is a minimal influence on the structure at the cut and a minimal temperature increase. The temperature increase is around $20^{\circ} \mathrm{C}$ and a small amount of water evaporation occurs, and sparks form around the beam. For some materials even this small increase in temperature can also mean a deterioration of the properties and possible destruction of the material.

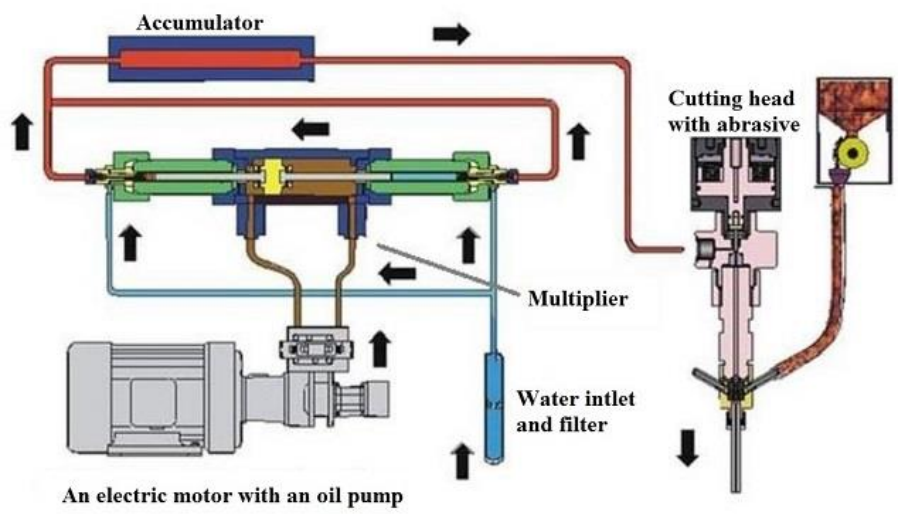

Fig. 1. Schematic of water jet device

\section{Water jet machining}

Cutting with pure water without adding abrasives involves the removal of material by the mechanical effect of the waterjet, which falls on the surface of the material at a high speed and high kinetic energy over a small surface area. Due to the absence of abrasive, there are higher demands on the working fluid, which is made up of water with certain required properties. Water seems to be the best choice for the working fluid, because it offers an acceptable compromise between the required properties.

In this method the beam is created in the cutting head. Water, which is compressed, passes through a nozzle with a small hole and is transformed into a thin waterjet with high kinetic energy and high speed. This beam impacts the machined material. After impact, the beam is decelerated due to the friction between the beam and the surface of the material. The pressure can reach values up to $690 \mathrm{MPa}$. The feed rate depends on the hardness, type and thickness of the material. The feed rate can be in the range of 5 to $400 \mathrm{~m} / \mathrm{min}$. The diameter of the nozzle is about $0.3 \mathrm{~mm}$, the beam has a diameter of 0.1 to $1.5 \mathrm{~mm}$. The width of the cut is slightly larger than the diameter of the nozzle (about $0.3 \mathrm{~mm}$ ). The distance of the cutting head from the material is from 2.5 to $6.35 \mathrm{~mm}$ but can also be 10 to $25 \mathrm{~mm}$. Pure waterjet technology is suitable for cutting soft, thin, non-rigid materials. It is possible to cut various plastic materials, rubber, plasterboard, foam materials, wood and plywood. It can also be used to remove corrosion or old coatings from products and devices. But a pure waterjet is not suitable for cutting metallic materials, because there is small removal of the material and it is more advantageous to use one of the abrasive methods where efficiency is higher. [1]

\section{Abrasive waterjet}

AWJ stands for abrasive waterjet and it is a method with a direct supply of abrasive. This technology works on the same principle as WJM with the material removed by the effects of a thin concentrated beam acting on a very small area. Because of the abrasive, the impact from AWJ has a much more mechanical effect. In this method, the abrasive is guided from the storage, which is outside the cutting device, straight into the mixing chamber in the cutting head. The flow of the beam causes mixing under-pressure in the chamber. This under-pressure causes suction, capture and acceleration of each abrasive particle. The dosage of the abrasive in this method is about 1 to $20 \mathrm{~kg} / \mathrm{min}$. Mixing power depends on the geometry of the focusing nozzle. The working pressure is in the range of 250 to $400 \mathrm{MPa}$. The diameter of the mixed beam is higher than the pure water one because of the abrasive. [2]

The feed rate depends on the cutting material. On hard materials, the feed rate is from $10 \mathrm{~mm} / \mathrm{min}$. Hard materials include cemented carbide, titanium and cobalt. Softer materials (aluminium, glass) can be cut with speeds up to 2500 $\mathrm{mm} / \mathrm{min}$. Mixing of the waterjet and the abrasive causes a deceleration of the beam by up to half of the original value, because it causes reduced performance. The selected abrasive must have a smaller particle size than the diameter of the hole in the focusing nozzle. The size of the abrasive should be at least five times smaller than the nozzle diameter. The smaller the hole in the nozzle, the more energy of the beam can be concentrated on one place. [3] 


\section{The experiment}

Samples which were cut using AWJ were documented and compared. Ten different conditions and modes were created, in which identical parts were cut. Only the feed rates were changed. The abrasive flow rate was not changed because the dosing is selected by software which chooses the appropriate conditions according to the thickness and type of the material to be cut. Additionally, these changes would be hard to observe on the final surface. During the experiment the feed rate, type of bullet holes and other support technologies were changed. [4]

\subsection{Samples}

The samples were rectangular, with dimensions $100 \mathrm{x} 40 \mathrm{~mm}$. A $12 \mathrm{~mm}$ thick sheet was selected as a blank. At this thickness the beam delays and surface quality changes could be easily observed. The tested material was an aluminiummagnesium alloy EN AW-5754 (AlMg3). Sheets of this material are available in a wide range of thicknesses, accessible and have good mechanical properties and good weldability. This means it is widely used in industry, especially for lightweight aluminium constructions. For this reason, EN AW-5754 was the ideal choice for the cut samples. A hole with a radius $\mathrm{R} 10$ was made on one edge of the sample, and on the other edge there was a bevel $10 \times 45^{\circ}$, to observe the surface when the trajectory is changed, other than $90^{\circ}$. For these reasons the sample also included a series of holes with diameter $10 \mathrm{~mm}$. The theoretical weight of the sample is 115 grams. [5]

The experiment took place on a device which consists of an X-Y CNC table with working dimensions $3000 \mathrm{x} 1500$ $\mathrm{mm}$, with abrasive dispensers and a JETS high pressure pump. The most important parameters of this pump are shown in the following table 1 .

\begin{tabular}{|c|c|}
\hline Parameter & Value \\
\hline Ambient temperature & $+5^{\circ} \mathrm{C}$ to $35^{\circ} \mathrm{C}$ \\
\hline Device dimensions & $1690 \times 1350 \times 1550 \mathrm{~mm}$ \\
\hline Weight & $1800 \mathrm{~kg}$ \\
\hline Max. outlet water pressure & $4100 \mathrm{bar}$ \\
\hline Max. amount of water for cutting & $3.81 / \mathrm{min}$ \\
\hline Max. total water consumption & $3.81 / \mathrm{min}$ \\
\hline Inlet water pressure & $3.5-6 \mathrm{bar}$ \\
\hline Max total input power & $39.3 \mathrm{~kW}$ \\
\hline Electric motor & $37 \mathrm{~kW} ; 1500 \mathrm{rpm}$ \\
\hline Hydraulic oil filling & 1601 \\
\hline Max. working oil pressure & $215 \mathrm{bar}$ \\
\hline Max. working oil temperature & $+52^{\circ} \mathrm{C}$ \\
\hline
\end{tabular}

Table 1. Parameters of high pressure pump

\subsection{The state of each sample}

\section{Sample 1}

Parameters: static shot, medium cut quality, speed $458.84 \mathrm{~mm} / \mathrm{min}$, DRC control off and Progress Jet on (Figure 2)

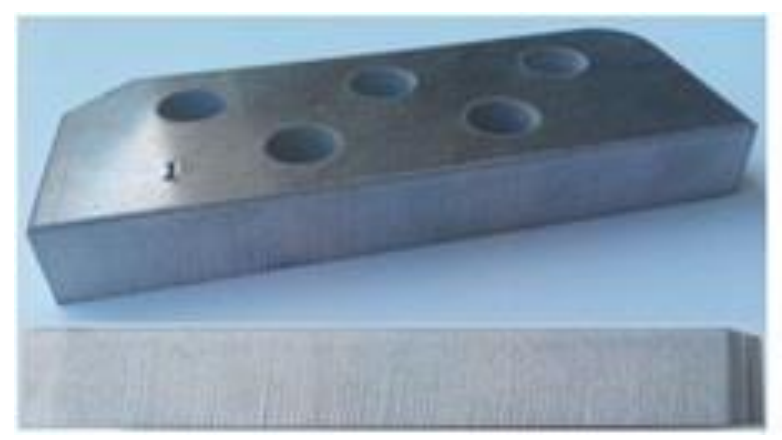

Fig. 2. Sample 1

\section{Sample 2}

Parameters: direct shot, medium cut quality, speed $458.84 \mathrm{~mm} / \mathrm{min}$, DRC control off and Progress Jet on (Figure 3) 


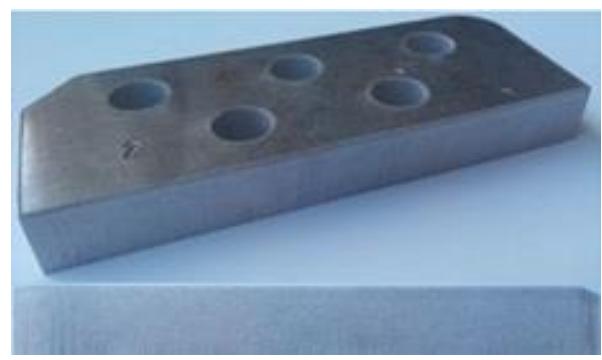

Fig. 3. Sample 2

\section{Sample 3}

Parameters: circular shot, medium cut quality, speed $458.84 \mathrm{~mm} / \mathrm{min}$, DRC control off and Progress Jet on (Figure 4)

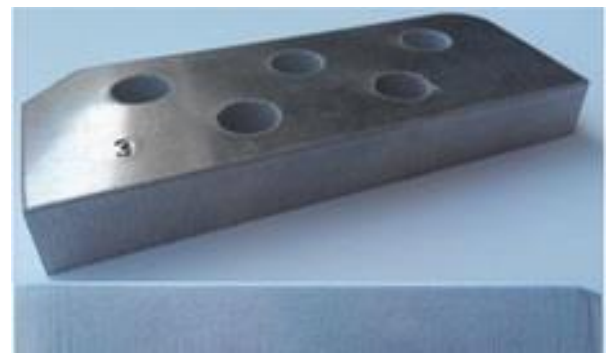

Fig. 4. Sample 3

\section{Sample 4}

Parameters: circular shot, the roughest cut quality, speed $458.84 \mathrm{~mm} / \mathrm{min}$, DRC control off and Progress Jet on (Figure 5)

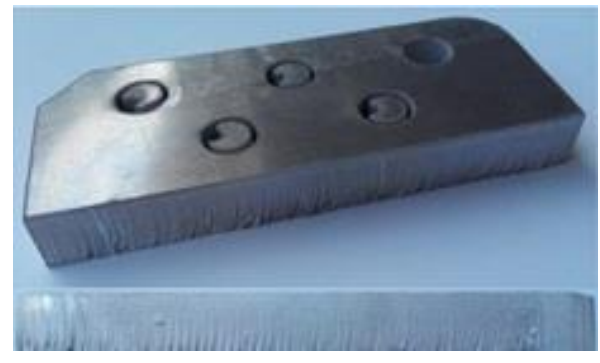

Fig. 5. Sample 4

\section{Sample 5}

Parameters: circular shot, rough cut quality, speed $731.43 \mathrm{~mm} / \mathrm{min}$, DRC control off and Progress Jet on (Figure 6)

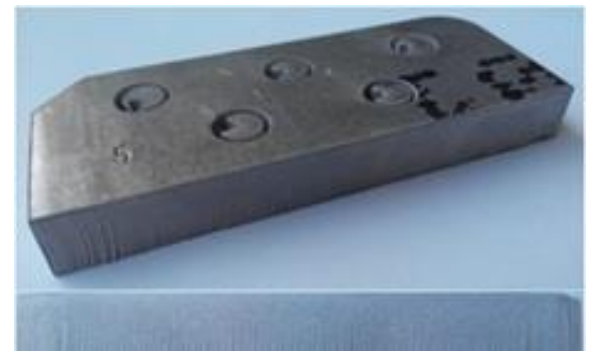

Fig. 6. Sample 5

\section{Sample 6}

Parameters: circular shot, medium cut quality, speed $458.84 \mathrm{~mm} / \mathrm{min}$, DRC control off and Progress Jet on (Figure 7) 


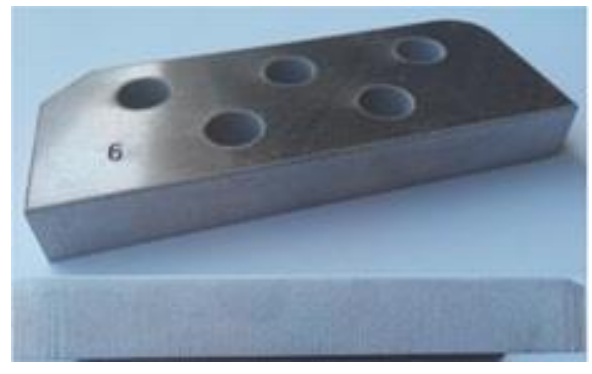

Fig. 7. Sample 6

\section{Sample 7}

Parameters: circular shot, smooth cut quality, speed $329.6 \mathrm{~mm} / \mathrm{min}$, DRC control off and Progress Jet on (Figure 8)

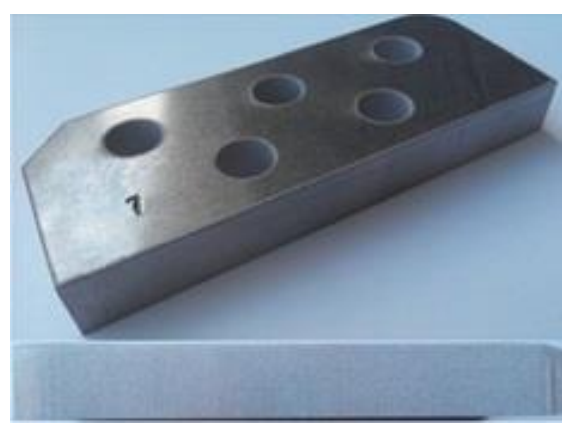

\section{Sample 8}

Fig. 8. Sample 7

Parameters: circular shot, the smoothest cut quality, speed $255 \mathrm{~mm} / \mathrm{min}$, DRC control off and Progress Jet on (Figure 9)

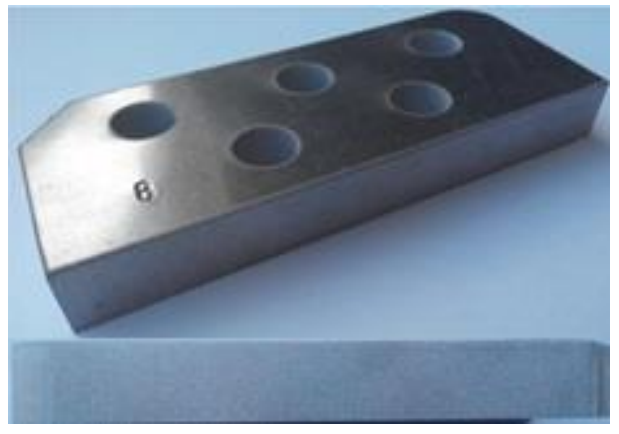

\section{Sample 9}

Fig. 9. Sample 8

Parameters: circular shot, smooth cut quality, speed $329.6 \mathrm{~mm} / \mathrm{min}$, DRC control on and Progress Jet on (Figure 10)

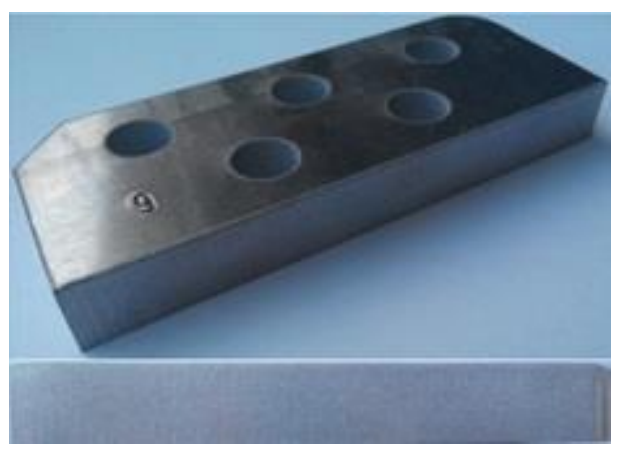

Fig. 10. Sample 9 


\section{Sample 10}

Parameters: circular shot, smooth cut quality, speed $329.6 \mathrm{~mm} / \mathrm{min}$, DRC control off and Progress Jet on (Figure 11)

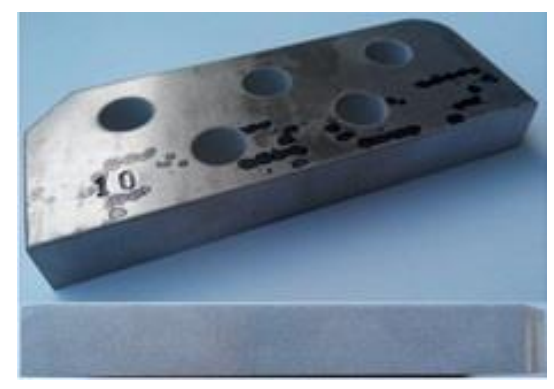

Fig. 11. Sample 10

\subsection{Cutting parameters}

Different setting properties were used for cutting the samples. Abrasive dosing settings were kept at $350 \mathrm{~g} / \mathrm{min}$ for all samples. The same setting was used for the first three samples, the only difference was the type of the bullet hole: static, straight and circular. The abrasive dosing was set at $200 \mathrm{~g} / \mathrm{min}$ for each bullet hole. Other samples remained with a circular bullet hole, which is the most suitable for most applications and the cutting speed was changed (samples 4 to 8). Speeds used are assigned to a particular cutting mode in the control system, the roughest, rough, medium, smooth and the smoothest. DRC controlling (radius correction) was used on Sample 9, and on sample 10 Progress Jet technology was turned off. Both the latest samples were cut in "smooth mode". A summary is shown in the following table (Table 2.)

\begin{tabular}{|c|c|c|c|c|c|}
\hline Sample & Bullet hole & Quality of cut & $\begin{array}{c}\text { Speed rate } \\
{[\mathbf{m m} / \mathbf{m i n}]}\end{array}$ & $\begin{array}{c}\text { DRC } \\
\text { controlling }\end{array}$ & Progress Jet \\
\hline 1 & static & medium & 458.84 & no & yes \\
\hline 2 & direct & medium & 458.84 & no & yes \\
\hline 3 & circular & medium & 458.84 & no & yes \\
\hline 4 & circular & coarsest & 1102.32 & no & yes \\
\hline 5 & circular & coarse & 731.43 & no & yes \\
\hline 6 & circular & medium & 458.84 & no & yes \\
\hline 7 & circular & smooth & 329.6 & no & yes \\
\hline 8 & circular & smoothest & 255 & no & yes \\
\hline 9 & circular & smooth & 329.6 & yes & yes \\
\hline 10 & circular & smooth & 329.6 & no & no \\
\hline
\end{tabular}

Table 2. Parameters of cutting

\subsection{Comparison in terms of the bullet hole}

Different types of bullet hole through material were used for samples 1 to 3 . The cutting mode was the same for samples with medium quality and therefore all the edges look practically the same. The first sample (static shot) has a distinct edge (mesh) across the whole depth. A substantially smaller edge was created on the second sample, which is only at the bottom of the sample depth. The third sample also has a small edge, but the area around the shot was cleaner. Therefore it appeared that the best and in practice the most used type of shot for thick walled sheet metal is the circular one. [4]

For samples 4 to 8, the circular type of shot was kept. The Progress Jet function was also enabled and DRC controlling was turned off. Only the speed mode was changed on these samples. On sample 4 the setting for the fastest cut was used, and therefore the worst quality of the cut surface was achieved. Exactly the opposite setting was used on sample 8, the slowest cut with best quality. The differences between each mode are very striking. The differences with changing speed were very noticeable on the samples. At higher speeds, in the rough and roughest modes, beam delays associated with the groove phenomenon can be observed. Sample 6 was cut with the middle mode, where the beam delay was less pronounced. [6]

The assumption was confirmed that with increasing feed rate there is a delay to the waterjet beam, which is subsequently shown on the grooves on the cut edge of the material. The result is that a higher feed rate means worse surface quality. The theoretical assumption was verified experimentally. It is interesting to note that on sample 4 the speed was so high that at several places on the cutting edge, complete cutting through the material did not occur. The sample had to be released from the blank using a hammer. [7] 


\section{Conclusion}

It has been concluded from these experiments that waterjet feed rate may have an impact on cut quality surface. After evaluating the cut samples, the circular shot appears to the best type of shot material, because it had the least effect on the relief of its surroundings. On the contrary, the worst type of shot was static shot, where a significant edge remained throughout the whole depth of the sample. From a qualitative standpoint, direct shot is located between these shots. In practice, the most commonly used material is circular shot because of its better properties. While cutting samples on which the feed rate was changed, it could easily be seen how with increasing speed, the quality of the cut surfaces decreases. Delaying of the waterjet beam was seen at the edges, which increases with increasing feed rate. According to expectations, higher speeds mean more beam delay and, at the end, worse cut quality. This bad trait of the waterjet had a great impact on the bevel, where the cutting head did not slow down as on the sharp edges and the result is poor surface quality and incomplete cutting of the material. On the sample with the highest feed rate (sample 4), there were several uncut locations, which actually caused great problems when removing the sample from the blank. Slightly uncut parts often occur even at slower speeds, but cut samples can usually be removed without any problems or much effort from the blank. This experiment provided a practical verification of the theoretical assumption. The practical part with the cut samples should serve mainly to provide a better idea and easier understanding of this technology. This experiment also shows that it is necessary to pay attention to ensuring the correct cutting parameters for a specific application, because even here can be seen the eternal duel between quantity versus quality. [8,9]

In the future we will focus on optimizing cutting conditions, different process parameters and their influence on the process.

\section{Acknowledgements}

This paper is based upon work sponsored by project SGS-2016-005

\section{References}

[1] Aišman, D. Staňková, H. Skálová, L. Mašek, B. (2008). Testing of the parameters of the Q-P process in high strength low-alloyed steel. In Danube Adria Association for Automation and Manufacturing. Vienna Austria: DAAAM International, Vienna Austria, 2008. ISBN: 978-3-901509-68-1, Vienna, Austria

[2] Jenicek, S. Kotesovec, V. Kalina, T. \& Masek, B. Use of Waterjet in Manufacturing Test Bars of High-Strength Steels, Annals of DAAAM for 2016 \& Proceedings of the 27th DAAAM International Symposium, pp.0219-0224, B. Katalinic (Ed.), Published by DAAAM International, ISBN 978-3-902734-08-2, ISSN 1726-9679, Vienna, Austria

[3] L.M. Hlavac, I.M. Hlavacova, L. Gembalova, J. Kalicinsky, S. Fabian, J. Mestanek, J. Kmec, V. Madr, Experimental method investigation of the abrasive water jet cutting quality, Journal of Materials Processing Technology 209 (2009) 6190-6195.

[4] Fabian Lissek, Michal Kaufeld, Jacqueline Tegas, Sergej Hloch, Online-monitoring for Abrasive Waterjet Cutting of CFRP via Acoustic Emission: Evaluation of Machining Parameters and Work Piece Quality Due to Burst Analysis, Procedia Engineering, Volume 149, 2016, Pages 67-76

[5] Kana, J. Vorel, I \& Ronesova, A. (2016). Simulator of Thermomechanical Treatment of Metals, Annals of DAAAM for 2015 \&Proceedings of the 26th DAAAM International Symposium, pp.0513-0518, B.Katalinic (Ed.), Published by DAAAM International, ISBN 978-3-902734-07-5, ISSN 1726-9679, Vienna, Austria

[6] Loeser, C. Duerr, H. \& Pilz, R. Application of the Micro Water Abrasive Injector Fine Jet for Precision Machining, Annals of DAAAM for 2010 \& Proceedings of the 21st International DAAAM Symposium, Volume 21, No. 1, ISSN 1726-9679 ISBN 978-3-901509-73-5, Editor B. Katalinic, Published by DAAAM International, Vienna, Austria, EU, 2010

[7] Yuvraj K. Madhukar, Suvradip Mullick, Ashish K. Nath. An investigation on co-axial water-jet assisted fiber laser cutting of metal sheets. Optics and Lasers in Engineering, Volume 77, February 2016, Pages 203-218

[8] D.S. Srinivasu, D.A. Axinte, Surface Integrity Analysis of Plain Waterjet Milled Advanced Engineering Composite Materials, Procedia CIRP, Volume 13, 2014, Pages 371-376

[9] Begic-Hajdarevic. D. Cekic. A. Mehmedovic. M. Djelmic. Experimental Study on Surface Roughness in Abrasive Water Jet Cutting 25th DAAAM International Symposium on Intelligent Manufacturing and Automation, DAAAM 2014 Experimental Study on Surface Roughness in Abrasive Water Jet Cutting, Procedia Engineering 100 ( 2015 ) $394-399$ 